\title{
Estrategias didácticas y motivacionales en las clases de educación física desde la teoría de la aułodeterminación
}

\section{David González-Cutre}

\section{Universidad Miguel Hernández de Elche Email: dgonzalez-cutre@umh.es}

RESUMEN: El objetivo de este artículo es explicar la didáctica de la educación física desde una perspectiva motivacional, tratando de ayudar al profesorado a promover la práctica autónoma de actividad física entre el alumnado. Para ello, en un primer apartado se exponen los conceptos establecidos por la teoría de la autodeterminación, mostrando la importancia de satisfacer las necesidades psicológicas básicas (autonomía, competencia y relación) de los estudiantes, para lograr formas de motivación más autodeterminadas (participar en educación física por disfrute, integrándola en el estilo de vida y considerándola importante) y consecuencias más positivas, como el aprendizaje o la adherencia a la práctica. En un segundo apartado se proporcionan estrategias motivacionales concretas, con ejemplos claros, para que el docente de educación física pueda satisfacer cada una de las necesidades psicológicas básicas de sus alumnos. Finalmente, se explica cómo el modelo de educación deportiva, como ejemplo de modelo de enseñanza que trata de unir el contexto educativo y de ocio, puede ayudar a satisfacer las necesidades psicológicas básicas. Este artículo pretende darle un nuevo enfoque a la didáctica de la educación física, facilitando la labor docente y convirtiéndose en un recurso práctico.

PALABRAS CLAVE: Didáctica, Motivación, Emoción, Transferencia.

\section{Teaching and motivational strategies in physical education classes from self-determination theory}

ABSTRACT: The objective of this article is to explain the physical education teaching from a motivational perspective, trying to help teachers promote students' autonomous physical activity. In the first section, I explain the concepts established by self-determination theory, showing the importance of satisfying students' basic psychological needs (autonomy, competence, and relatedness) to achieve more selfdetermined forms of motivation (participating in physical education for enjoyment, integrating it in lifestyle, and considering it important) and positive consequences, such as learning or adherence to physical activity. In the second section, I provide specific motivational strategies, with clear examples, for the physical education teacher to satisfy each 
basic psychological need of the students. Finally, I explain how the sport education model, as an example of a teaching model that tries to connect the educational and leisure contexts, can help to satisfy the basic psychological needs. This article hopes to give a new focus to the physical education teaching, facilitating the teacher work and becoming a practical resource.

KEY WORDS: Teaching, Motivation, Emotion, Transfer.

\section{LA DIDÁCTICA DE LA EDUCACIÓN FÍSICA ENTENDIDA DESDE UNA PERSPECTIVA MOTIVACIONAL}

Uno de los principales objetivos de las clases de educación física es el de la promoción de la práctica de actividad física en el alumnado. En esta sociedad cada vez más sedentaria, las clases de educación física juegan un papel muy importante, convirtiéndose en ocasiones en el único momento de la semana en el que el niño o adolescente practica deporte. La calidad de las experiencias vividas en la clase de educación física va a ser clave para promover el interés hacia la práctica deportiva y el deseo de ocupar el tiempo libre realizando esta actividad. A pesar de la reducida carga horaria de la educación física en el sistema educativo español, existen estudios que demuestran la relevancia del profesorado de educación física para la promoción de la actividad física en la infancia y la adolescencia (González-Cutre, Sicilia, Beas-Jiménez, y Hagger, 2014). Si en la clase de educación física se consigue que los estudiantes disfruten mientras adquieren un nivel básico de destreza motriz, es probable que continúen practicando en sus ratos libres. Sin embargo, si la clase de educación física se convierte en una experiencia traumática caracterizada por la ausencia de motivación y que pone de manifiesto la incompetencia motriz, será difícil que el niño o adolescente quiera comprometerse con una práctica física continuada (Beltrán-Carrillo, Devís-Devís, Peiró-Velert, y Brown, 2012). Dado que la literatura refleja claramente un efecto trans-contextual de que lo que ocurre en el aula va más allá del centro educativo (Hagger y Chatzisarantis, 2012), influyendo en la vida del estudiante, resulta de sumo interés la correcta formación del docente de educación física en estrategias didácticas y motivacionales que ayuden a la promoción de la actividad física.

Actualmente ha cobrado mucha importancia una vertiente de la investigación que trata de aplicar teorías motivacionales a la didáctica de la actividad física y del deporte (Moreno, Cervelló, González-Cutre, Julián, y Del Villar, 2011) para explicar los procesos básicos de transmisión de información inicial, feedback, organización y control del grupo, y aprovechamiento del tiempo de práctica. Bajo este paradigma motivacional, la teoría de la autodeterminación (Deci y Ryan, 2000; Ryan y Deci, $2000,2017)$ ha resultado ser un marco de referencia óptimo para explicar los procesos que acontecen en la clase de educación física, permitiéndonos entender el mecanismo que subyace a la relación entre la actuación del profesorado y las consecuencias de su actuación (Ntoumanis, 2012). Esta relación es explicada introduciendo el efecto mediador de las necesidades psicológicas básicas y la motivación, obteniendo la secuencia motivacional presentada en la Figura 1. 


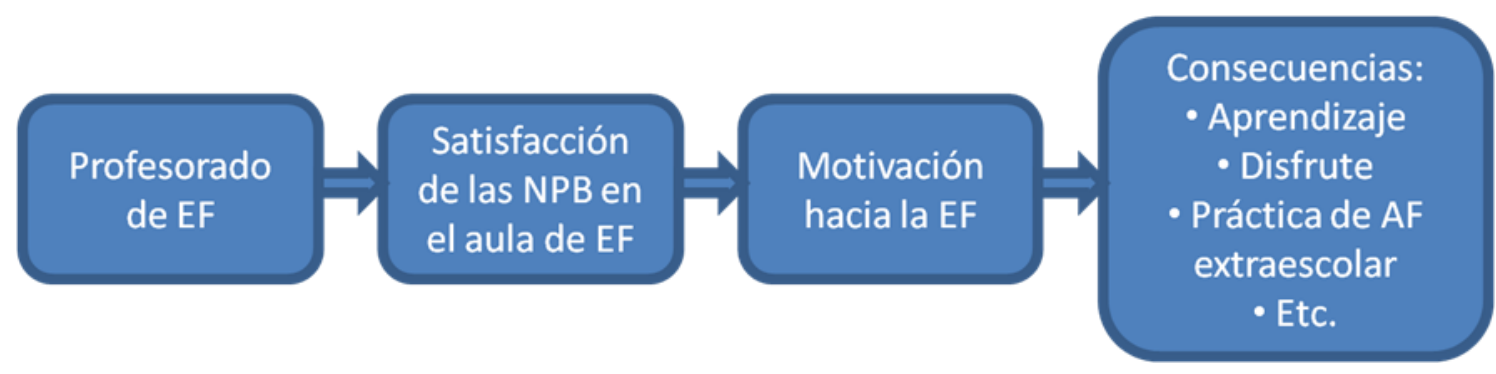

Figura 1. Secuencia motivacional de la teoría de la autodeterminación aplicada a la clase de educación física. (Nota. EF = Educación Física. NPB = Necesidades Psicológicas Básicas. AF = Actividad Física)

Atendiendo a la teoría de la autodeterminación, el profesorado de educación física desempeña un rol fundamental sobre la satisfacción/frustración de las necesidades psicológicas básicas (autonomía, competencia y relación) de los estudiantes, generando tipos de motivación más o menos autodeterminados (con un origen más interno o menos), que se van a manifestar en una serie de consecuencias como el aprendizaje de los elementos básicos de la educación física, el disfrute durante las clases y el deseo de seguir practicando actividad física al sobrepasar las puertas del centro escolar. Por ello, es imprescindible dotar al profesorado de educación física de estrategias concretas que ayuden a que los alumnos experimenten sentimientos de autonomía, competencia y relación durante sus clases. Éste va a ser el objetivo del presente artículo.

Antes de entrar de lleno en este objetivo, es necesario llevar a cabo una aclaración conceptual de los diferentes términos que establece la teoría de la autodeterminación, para poder entender mejor su secuencia motivacional y la aplicación de estrategias didácticas bajo esta perspectiva. El profesorado de educación física debe estar totalmente familiarizado con esta terminología para poder emplear con éxito las estrategias descritas más adelante.

La teoría de la autodeterminación postula que todas las personas tienen tres necesidades psicológicas básicas: autonomía, competencia y relación, que necesitan satisfacer en cualquier contexto en el que interactúan. Uno de los contextos en el que el ser humano pasa más tiempo, y que por tanto más influye en su desarrollo, es el contexto educativo, que se convierte en un arma muy poderosa representando un agente de cambio significativo. Aplicando estos conceptos a la clase de educación física, es de suma importancia conseguir que los alumnos experimenten el éxito en el mayor número de ocasiones posibles, de manera que perciban que están aprendiendo conceptos y habilidades y que van mejorando (competencia). Igualmente, bajo una concepción moderna del sistema educativo, se debe dotar al alumnado de autonomía, permitiéndole tomar decisiones y elegir cosas importantes dentro del proceso de enseñanza-aprendizaje. Por último, es necesario tener en cuenta que el ser humano es un ser social y, por tanto, el docente de educación física debe hacer el esfuerzo de promover las relaciones sociales en clase, más si cabe teniendo en cuenta que el movimiento, a través de diferentes tareas y juegos, tiene una gran potencial para ello.

La satisfacción de estas tres necesidades psicológicas básicas en el aula de educación física va a conducir a que el estudiante experimente formas de motivación más autodeterminadas (regulación identificada, integrada e intrínseca). La regulación identificada se caracteriza porque el alumno participa en la clase de educación física motivado por considerar importante el papel que juega la asignatura y lo que allí está aprendiendo; por ejemplo, porque aprende conceptos tácticos y desarrolla habilidades 
motrices que luego puede extrapolar a la práctica deportiva, o porque se da cuenta de la importancia que tiene para la salud practicar actividad física de forma regular, y aprende métodos de desarrollo de la condición física que puede utilizar en su tiempo libre. La regulación integrada supone un pasito más en la autodeterminación, consiguiendo integrar la actividad física en general, y la educación física en particular, en el estilo de vida, convirtiéndose la práctica de deporte en un pilar fundamental que es congruente con los valores que posee el estudiante en relación a la adquisición de un estilo de vida saludable (ya no sólo se considera la actividad importante sino que además es un reflejo de lo que uno es). La motivación intrínseca representa el mayor nivel de autodeterminación y tiene lugar cuando el alumnado participa en las clases porque se divierte con lo que hace, le gusta y le satisface.

Sin embargo, la insatisfacción o frustración de las necesidades psicológicas básicas en el aula de educación física conduce a formas de motivación más desadaptativas y no autodeterminadas (regulación introyectada, externa y desmotivación). Un estudiante motivado de forma introyectada sólo participará en las tareas planteadas por un sentimiento de culpabilidad y responsabilidad, sin obtener durante la práctica nada más interno que pueda valorar positivamente, lo que obviamente no se asocia a la perduración de la conducta. Lo mismo ocurre con la regulación externa, que representa el comportamiento motivado por la obligación del profesor o el sistema educativo para hacer algo, porque es lo que marcan las normas y se debe hacer para evitar castigos y represalias. Finalmente tenemos la desmotivación, que es el estado motivacional deprimido, caracterizado por un total desinterés y falta de implicación en el aula de educación física.

La literatura (para una revisión ver Vallerand, 2007) muestra que la satisfacción de las necesidades psicológicas básicas y el desarrollo de formas de motivación más autodeterminadas (identificada, integrada e intrínseca) en la clase de educación física, se relaciona con consecuencias más positivas en el alumnado: un mayor interés, emociones positivas, estados psicológicos óptimos caracterizados por un gran concentración, un mayor esfuerzo y rendimiento, y una mayor adherencia a la actividad física. Sin embargo, la insatisfacción o frustración de las necesidades y el desarrollo de formas de motivación menos autodeterminadas (introyectada, externa y desmotivación), se asocia con consecuencias más negativas como el aburrimiento, la infelicidad o el abandono de la práctica física.

En esta línea, y tomando como referencia la Figura 1, el profesorado de educación física deberá aplicar estrategias motivacionales para fomentar la satisfacción de las necesidades psicológicas básicas de autonomía, competencia y relación, generando tipos de motivación más autodeterminados que lleven a consecuencias más adaptativas, como la adherencia a la práctica de actividad física en el tiempo libre. Durante este artículo vamos a ir delineando con profundidad las diferentes estrategias que podrían ser aplicadas en la clase de educación física, dotando al profesorado de una guía práctica que le podría ayudar a ser más eficaz en su labor docente. Finalmente, se introduce brevemente el modelo de educación deportiva (Siedentop, 1994) y su relación con la satisfacción de las tres necesidades psicológicas básicas, como ejemplo de modelo de enseñanza que trata de unir el contexto educativo y de ocio. 


\section{ESTRATEGIAS DIDÁCTICAS Y MOTIVACIONALES APLICAR EN LA CLASE DE EDUCACIÓN FÍSICA}

Partiendo de trabajos previos (González-Cutre, Martínez-Camacho, GómezRijo, y Moreno, 2010; González-Cutre y Moreno, 2009; Moreno et al., 2011; Moreno y González-Cutre, 2006), y haciendo el esfuerzo de adaptarnos a la realidad concreta del aula de educación física, a continuación se proponen y se desarrollan en profundidad una serie de estrategias didácticas y motivacionales específicas, agrupadas por su influencia sobre cada una de las necesidades psicológicas básicas (Tabla 1):

Tabla 1. Estrategas motivacionales aplicadas al aula de educación física

\begin{tabular}{|c|c|c|}
\hline $\begin{array}{l}\text { Necesidades } \\
\text { psicológicas }\end{array}$ & Estrategias & Ejemplos \\
\hline \multirow[t]{11}{*}{ Competencia } & 1. Proporcionar suficiente tiempo & $\begin{array}{c}\text { 3-4 tareas por sesión. Mínimo 8-10 } \\
\text { sesiones por unidad didáctica }\end{array}$ \\
\hline & 2. Actividades novedosas & $\begin{array}{c}\text { BodyPump®, BodyCombat } \AA \text {, } \\
\text { CrossFit, Kinball, Zumba, Raids de } \\
\text { aventura, barranquismo, TICs: } \\
\text { Endomondo, Facebook }\end{array}$ \\
\hline & 3. Reglas para que todos sean partícipes & Tocar todos el balón \\
\hline & $\begin{array}{c}\text { 4. Importancia del esfuerzo y la } \\
\text { superación personal }\end{array}$ & $\begin{array}{c}\text { Evaluación significativa y continua } \\
\text { del proceso }\end{array}$ \\
\hline & 5. Reconocer el progreso individual & $\begin{array}{l}\text { No sólo hay que dar feedback } \\
\text { cuando la ejecución es incorrecta }\end{array}$ \\
\hline & 6. Feedback positivo & $\begin{array}{c}\text { Feedback prescriptivo, interrogativo } \\
\text { y afectivo. Tipo sándwich }\end{array}$ \\
\hline & 7. Convencer de que se puede mejorar & Creencia incremental de habilidad \\
\hline & 8. Utilizar la música & $\begin{array}{c}\text { Trabajando la resistencia a través } \\
\text { de la carrera, juegos deportivos, } \\
\text { expresión corporal }\end{array}$ \\
\hline & 9. Objetivos a corto plazo y adaptados & $\begin{array}{c}\text { Progresión. Estilos } \\
\text { individualizadores: Grupos de nivel, } \\
\text { Caladú } \\
\end{array}$ \\
\hline & 10. Implicar en las demostraciones & $\begin{array}{c}\text { No obligar, persuadir. Variar la } \\
\text { persona elegida }\end{array}$ \\
\hline & 11. Evitar agrupar según la competencia & $\begin{array}{c}\text { Tener cuidado con los } \\
\text { agrupamientos libres } \\
\end{array}$ \\
\hline \multirow[t]{5}{*}{ Autonomía } & 12. Dar posibilidad de elección & $\begin{array}{c}\text { Objetivos, contenidos, reglas, } \\
\text { espacio, tiempo, material, técnica } \\
\text { de enseñanza, normas, evaluación }\end{array}$ \\
\hline & 13. Explicar los objetivos didácticos & $\begin{array}{l}\text { En cada tarea. E.g., abdominales } \\
\text { por salud y estabilidad del tronco }\end{array}$ \\
\hline & $\begin{array}{l}\text { 14. No crear dependencia de las } \\
\text { recompensas }\end{array}$ & No dar feedback en cada ejecución \\
\hline & 15. Estilos participativos & $\begin{array}{c}\text { Enseñanza recíproca, grupos } \\
\text { reducidos, microenseñanza: Dirigir } \\
\text { calentamiento o sesión }\end{array}$ \\
\hline & 16. Estilos cognitivos y creativo & $\begin{array}{l}\text { Descubrimiento guiado, resolución } \\
\text { de problemas. Creatividad: } \\
\text { Representar elementos de una } \\
\text { hoja: belén, zoo, fórmula uno, } \\
\text { cuarto de baño }\end{array}$ \\
\hline
\end{tabular}




\begin{tabular}{|c|c|c|}
\hline \multirow[t]{3}{*}{$\begin{array}{l}\text { Relación } \\
\text { entre } \\
\text { alumnos }\end{array}$} & 17. Fomentar la interacción & $\begin{array}{c}\text { Estilo socializador: Objetivo común, } \\
\text { todos necesarios, cambios de } \\
\text { grupo (baraja española) }\end{array}$ \\
\hline & $\begin{array}{l}\text { 18. Agrupaciones flexibles y } \\
\text { heterogéneas }\end{array}$ & $\begin{array}{l}\text { Aleatorios, equilibrados, } \\
\text { aprendizaje cooperativo }\end{array}$ \\
\hline & $\begin{array}{l}\text { 19. Evitar rivalidad y desarrollar } \\
\text { autocontrol }\end{array}$ & $\begin{array}{c}\text { Utilizar el símil de la lata de coca- } \\
\text { cola para prevenir problemas entre } \\
\text { los estudiantes }\end{array}$ \\
\hline \multirow[t]{3}{*}{$\begin{array}{l}\text { Relación } \\
\text { profesor- } \\
\text { alumno }\end{array}$} & $\begin{array}{l}\text { 20. Clima de preocupación e inteligencia } \\
\text { emocional }\end{array}$ & $\begin{array}{c}\text { Interesarse por la vida del alumno, } \\
\text { tratar por el nombre, percibir y } \\
\text { transmitir emociones }\end{array}$ \\
\hline & 21. Pasión & Contagiar la pasión, sonreír \\
\hline & 22. Importancia de cada sesión & $\begin{array}{c}\text { No pagar los problemas personales } \\
\text { con el alumnado }\end{array}$ \\
\hline
\end{tabular}

\subsection{Estrategias para fomentar la competencia:}

Proporcionar suficiente tiempo para realizar las tareas: Pensemos que ocurriría si en nuestra vida diaria alguien nos propusiera un acertijo, jeroglífico o adivinanza, y antes de que nos diera tiempo a resolverlo nos dijera la solución, o peor aún, nos quitara el jeroglífico y nunca nos dijera la solución. O vayamos más allá, a un contexto laboral donde nuestro jefe nos pide que elaboremos un informe importante de la noche a la mañana, y finalmente no tenemos tiempo de terminarlo para la hora de la reunión. Sentiríamos que no nos han dado tiempo suficiente para desarrollar aquella tarea encomendada, aflorando un sentimiento de incompetencia. Esta idea es fácilmente extrapolable a las clases de educación física, a dos niveles: sesión y unidad didáctica.

En primer lugar, es importante que el profesor de educación física planifique bien las sesiones para que al alumnado le dé tiempo a conseguir lo que se le pide. Por un lado, con tres o cuatro tareas por sesión suele ser suficiente para conseguir los objetivos previstos, así que el docente no debe obsesionarse con plantear muchas tareas, ya que, además de abusar del tiempo de organización, el alumnado no va a ser capaz de experimentar el éxito. Por otro lado, es necesario que el docente sea flexible con la planificación inicial y ejerza una labor de reflexión durante la práctica, observando el desarrollo de las tareas y proporcionando más tiempo si el estudiante lo necesita.

En segundo lugar, a nivel de la unidad didáctica, es fundamental programar unidades didácticas lo suficientemente largas para desarrollar los contenidos planteados. Últimamente está de moda la programación de muchas unidades didácticas de corta duración para que el alumnado tenga un amplio repertorio de posibilidades de actividad física, pero bajo el punto de vista de esta estrategia recomendamos reducir el número de unidades didácticas anuales, de manera que cada una de ellas desarrolle un mínimo de 8-10 sesiones para que el alumnado tenga tiempo de aprender ese contenido.

Desarrollar actividades novedosas: Podría parecer que plantear actividades nuevas, que el alumnado no conoce $y$, por lo tanto, no sabe realizar correctamente, va a provocar un sentimiento de incompetencia. No obstante, si atendemos a la estrategia anterior e introducimos los nuevos contenidos de forma progresiva, proporcionando tiempo para su asimilación, la novedad se convierte en un elemento motivador imprescindible en las clases de educación física. La búsqueda de novedad tiene un carácter innato y universal, dado que, desde el nacimiento, los niños en cualquier cultura son activos, inquisitivos y curiosos, necesitando una cierta cantidad de 
estimulación nueva (Deci y Ryan, 1985). De hecho, una investigación reciente propone que la novedad podría ser la cuarta necesidad psicológica básica (González-Cutre, Sicilia, Sierra, Ferriz, y Hagger, 2016). En este sentido, es interesante que el profesorado de educación física se renueve y se adapte a la realidad del momento, incluyendo nuevos contenidos en las clases de educación física.

Por ejemplo, actualmente en secundaria, dentro del bloque de condición física y salud, sería interesante incluir actividades dirigidas para el desarrollo de la fuerza y la resistencia, como algunas variantes del BodyPump®, el BodyCombat ${ }^{\circledR}$, o el CrossFit; o aprovechar el creciente desarrollo del triatlón para despertar el interés respecto al entrenamiento de las cualidades físicas; o incorporar una unidad didáctica de comba como medio de entrenamiento; dentro del bloque de juegos y deportes se podrían incluir juegos tradicionales que el alumnado desconociera o juegos alternativos como el Kinball o el Colpbol, así como aplicar el modelo de educación deportiva; dentro del bloque de expresión corporal incluir contenidos como la Zumba, bailes latinos o el Acrosport; también se debería dar una mayor importancia al bloque de actividades en medio natural, al tratarse de actividades que el estudiante puede hacer en su entorno próximo y que además están en auge: carreras por montaña, raids de aventura, escalada, descenso de barrancos, Geocaching, etc.

Por último, resulta de interés incorporar las tecnologías de la información y la comunicación (TICs), dada su creciente evolución en los últimos años. Algunos ejemplos de aplicación de las TICs en clases de educación física podrían ser: Utilizar alguna tablet para que el alumnado registre comportamientos durante una observación; hacer uso de programas de seguimiento GPS, como el Endomondo o el Runtastic, para que el alumnado sepa la distancia recorrida y vea la ruta realizada; usar las redes sociales (por ejemplo un grupo de Facebook) para compartir con los estudiantes información relevante relacionada con la importancia y beneficios de la actividad física, dándoles consejos y sugerencias para su participación de forma autónoma.

Establecer reglas para que todos se sientan partícipes: A la hora de diseñar tareas, el docente de educación física debe tratar de establecer normas que faciliten la participación en condiciones similares, de tal manera que todos los estudiantes tengan la oportunidad de experimentar el éxito. El ejemplo más típico lo representa la introducción, en cualquier juego deportivo colectivo, de la regla de que el balón debe pasar por todos los componentes del equipo antes de poder anotar un tanto. Resulta interesante explicar el porqué de esta regla a los alumnos, haciéndoles ver que todos deben tener posibilidades de aprender en ese entorno educativo. Otros ejemplos de actividades en las que todos se pueden sentir partícipes, pues tienen un rol asignado durante la práctica, pueden ser la realización de una coreografía grupal de expresión corporal, una carrera de relevos en la que hay que transportar cada vez a un miembro del grupo en el aire entre todos los compañeros, o un desplazamiento coordinado en tríos sobre unos esquís de fondo construidos por los propios alumnos con material cotidiano.

Incidir en la importancia del esfuerzo y la superación personal: Como ya bien nos indicaba la teoría de las metas de logro (Nicholls, 1989), es importante que el profesor de educación física priorice en el alumnado un sentimiento de competencia ante el progreso personal y la realización adecuada de las tareas a través del esfuerzo. Se debe huir de concepciones clásicas del aula de educación física basada en el rendimiento, en las cuales unos pocos más competentes marcaban los criterios de éxito que todos debían alcanzar y que se manifestaban en unos baremos establecidos por el profesorado de educación física. Superar a los demás no lo puede conseguir todo el mundo, pero superarse a sí mismo sí. Por tanto, el docente de educación física 
debe reconocer cuando un alumno mejora y decírselo de forma explícita. Igualmente, la evaluación tiene que ser una fuente de aprendizaje significativo, de manera que el alumnado sepa qué cosas ha hecho bien y cuáles debe mejorar. Sería interesante incorporar un sistema de evaluación continua que permita registrar determinados comportamientos y actitudes del alumnado, viendo la evolución en el proceso, y no sólo la adquisición de un producto final.

Por otro lado, centrarse en la superación personal no supone ni mucho menos la eliminación de la competición de las clases de educación física. Vivimos en una sociedad competitiva y debemos educar al niño y adolescente para que pueda adaptarse a ella. Lo importante es darle una orientación educativa a esa competición, reconociendo a los ganadores (pues todos necesitamos que nos refuercen nuestros logros) pero mostrado que hay otros elementos a considerar dentro de esa competición, como la implicación, el disfrute, el aprendizaje o la adquisición de valores.

Reconocer el progreso individual: En la mayoría de ocasiones el profesorado de educación física suele utilizar el feedback para corregir al alumno ante una actuación que no ha sido óptima. Sin embargo, es también importante utilizar el refuerzo externo para señalar las mejoras y los logros obtenidos. El propio estudiante a veces no es capaz de darse cuenta de lo que ha conseguido y el docente debe ayudarle a ello. El juicio emitido por una persona experta es un referente que motiva al alumnado a esforzarse en la realización de la tarea.

Suministrar un feedback positivo: En línea con la anterior estrategia, resulta clave la forma en que el docente transmite la información de corrección al alumno. No es lo mismo decir en una tarea de balonmano: “¿Eso es un pase? ¿Con esa fuerza?”, que decir: "Ha sido un buen intento al ver a tu compañero desmarcado, pero el pase se ha quedado un poco corto. La próxima vez trata de armar el brazo un poco más hacia atrás y continuar con el movimiento una vez sueltes el balón, para imprimirle algo más de fuerza. Ánimo, que seguro que lo consigues". Se debe utilizar por tanto el feedback prescriptivo, diciéndole al alumno cómo corregir su acción (o bien interrogativo para que se dé cuenta él mismo), combinado con el feedback afectivo. La estrategia tipo sándwich es el ejemplo más usual de feedback positivo. La rebanada de arriba sería lo que se ha hecho bien, el embutido sería lo que ha fallado, y la rebanada de abajo sería la frase de ánimo y de confianza en que puede lograr hacerlo bien.

Convencer al alumno de que siempre se puede mejorar: La literatura describe dos creencias en cuanto a la habilidad (Dweck, 1999). Existen estudiantes que creen que su habilidad es estable y dependiente del talento natural, y que por mucho que se esfuercen no van a conseguir mejorar (creencia de entidad). Otros, sin embargo, consideran que si se esfuerzan y trabajan duro seguro que lo van a hacer cada vez mejor (creencia incremental). Dada la heterogeneidad del aula de educación física, es frecuente encontrarse con alumnos que reflejan una creencia de entidad y que lo manifiestan con frases del tipo: "Profesor no me sale. Yo no puedo hacer esto. No sirvo para esto". Como creencias que son, es labor del docente cambiarlas. La utilización de las estrategias relacionadas con la competencia, aquí descritas, son el primer paso para ello. El segundo, decirle al estudiante que está equivocado y mostrarle todo lo que está aprendiendo.

Utilizar la música en la clase de educación física: Aunque no se debe abusar de ella, pues perdería su carácter sorpresivo y novedoso, resulta de interés la incorporación de la música en las sesiones de educación física. Hay estudios que demuestran el efecto motivacional de la música, generando un cambio en la actitud y en la autoevaluación, a través de la mejora de la competencia percibida y el desarrollo de un sentimiento de control (Karageorghis, 2017; Priest y Karageorghis, 2008). Esto 
incide además en una mayor persistencia, nivel de trabajo y adherencia a la práctica. Por ejemplo, se puede utilizar la música cuando se está trabajando la resistencia a través de la carrera (emulando una situación cotidiana como puede ser correr con un Mp3), o cuando se realizan juegos deportivos competitivos. Por supuesto, juega un papel imprescindible en el bloque de expresión corporal.

Establecer objetivos a corto plazo y adaptados al nivel de alumno: Por un lado, es necesario que el alumno perciba que va mejorando poco a poco. Aquí es muy relevante el concepto de progresión de aprendizaje. Se deben parcelar los objetivos finales en pequeños objetivos que el alumnado debe poder asimilar. Dentro de una misma sesión podemos ir de los más simple a lo más complejo. Por ejemplo, en una sesión donde se trabaje el control y el pase en fútbol, podemos empezar con una situación más estática de rondos donde el jugador de en medio trata de robar el balón, evolucionando a una situación de juego modificada con varias porterías, para acabar con una situación de juego que mantenga las reglas del deporte original. Del mismo modo, dentro de una unidad didáctica, debemos dedicar unas sesiones al aprendizaje de los elementos básicos para, posteriormente, cuando el alumnado perciba que controla dichos elementos, plantear situaciones más aplicadas. Por ejemplo, en una unidad didáctica de actividades gimnásticas, se pueden emplear las primeras sesiones para el aprendizaje de algunos elementos gimnásticos individuales y figuras grupales, para posteriormente construir una coreografía aplicando lo aprendido.

Respecto a la adaptación al nivel del estudiante, ésta es una de las grandes batallas del profesorado. Por una parte, se deben realizar pequeñas adaptaciones de las tareas para facilitar la ejecución (por ejemplo, permitir a una persona dar más toques al balón en un rondo antes de pasarle a su compañero). Por otra parte, el uso de estilos individualizadores (Delgado Noguera, 1991) en las clases de educación física resulta de sumo interés. Para la aplicación de estos estilos es clave explicarle al alumnado el porqué de esta manera de trabajar, de forma que entienda que los alumnos de más nivel van a poder avanzar más rápido y los de menos nivel van a poder llegar más lejos. Así se trata de evitar la concepción por parte del aula de una división clasista de buenos y malos. No obstante, no se deben utilizar estos estilos individualizadores durante mucho tiempo, ya que priorizan la competencia motriz sobre otros aspectos sociales que también interesa desarrollar. Continuando con el ejemplo de la unidad didáctica de actividades gimnásticas, y teniendo en cuenta la disparidad de niveles respecto a este contenido en el aula de educación física, podría ser útil establecer dos grupos de nivel, empezando uno de ellos a trabajar por el volteo adelante hasta llegar a la rueda lateral, y otro empezando por la rueda lateral hasta llegar a la paloma de manos. Poniendo otro ejemplo dentro del bloque de condición física y salud, se ha mostrado muy útil el software Caladú (Generelo, Julián, y Zaragoza, 2009) como aplicación de la enseñanza programada (programas individuales informatizados) para una unidad didáctica de carrera de larga duración. El estudiante realiza un test que consiste en correr la máxima distancia posible en cinco minutos, y a partir de la distancia conseguida el programa diseña un plan de entrenamiento personalizado que es desarrollado en la clase de educación física.

Implicar al alumno en la realización de demostraciones: A veces es frecuente utilizar al estudiante para hacer una demostración de una determinada ejecución motriz. Aunque utilizar al alumnado puede ser un buen recurso para mostrar que la habilidad la puede desarrollar cualquiera, también se puede convertir en un elemento conflictivo. Por un lado, es importante preguntarle al alumno si le apetece hacer la demostración, no obligándole a hacer algo con lo que puede quedar en ridículo delante de todos sus compañeros. Ante la ausencia de voluntarios o la negatividad de un estudiante a hacer una demostración, debemos utilizar la persuasión para que se dé cuenta de que no va a pasar nada e intentar motivarle a la acción, pero sin obligarle. 
Por otro lado, el docente debe ir variando la persona que utiliza para hacer la demostración, de tal manera que todos se puedan sentir importantes y se evite la estigmatización del más capaz ("el enchufado") o del menos capaz ("el inútil").

Evitar agrupar al alumnado siguiendo criterios de competencia: Aunque hemos comentado que en ocasiones resultan de utilidad los estilos individualizadores, con una finalidad didáctica clara que el docente debe explicar a los alumnos, lo cierto es que en el resto de ocasiones el profesor debe evitar que los agrupamientos pongan de manifiesto quiénes son los estudiantes más incompetentes. El ejemplo más claro se da cuando el docente deja al alumnado que elabore los grupos libremente, de manera que dos estudiantes van pidiendo consecutivamente a sus compañeros para conformar dos grupos, quedándose las personas con menor nivel de habilidad en último lugar todas las veces. De esta manera se genera un sentimiento de incompetencia aprendida que es fácilmente evitable utilizando otros sistemas de agrupación, como el aleatorio (e.g., asignado números) o el de formación de equipos equilibrados por parte del docente. Sirva también de ejemplo de lo que no se debe hacer, un video que corre por las redes sociales donde un niño sudamericano algo "gordito" participa en una carrera de ida y vuelta a lo largo de una pista con sus compañeros en educación física. Nada más comenzar la carrera el niño "gordito" se queda en última posición, con mucha diferencia, y aunque hace trampas y da la vuelta mucho antes que todos sus compañeros, de nuevo vuelve a ser rebasado y acaba en última posición. La profesora decide formar una fila siguiendo las posiciones obtenidas, de manera que Angelito (el niño "gordito") queda relegado al último puesto. A pesar de que había intentado colocarse en el octavo puesto en la fila, la profesora se encarga de quitarle de ahí y recordarle que ha sido el último.

\subsection{Estrategias para fomentar la autonomía:}

Dar posibilidad de elección y fomentar la participación en el proceso de toma de decisiones: Existen infinidad de cosas que el docente de educación física puede dar a elegir al alumnado, permitiendo su participación en el proceso. A continuación ponemos algunos ejemplos:

1) Objetivos: Hemos comentado previamente que para fomentar la percepción de competencia es necesario establecer objetivos adaptados al alumno. Otra estrategia para ello, aparte de las mencionadas en el apartado anterior, puede ser dar a elegir esos objetivos al propio alumnado. Por ejemplo, dentro de una sesión de acondicionamiento de la musculatura del tronco, podemos establecer un mínimo de 15 y un máximo de 30 repeticiones de cada ejercicio, de manera que los alumnos se ajusten a sus posibilidades, consiguiendo así la satisfacción de las necesidades de competencia y autonomía.

2) Contenidos: Se pueden plantear contenidos o tareas que persigan los mismos objetivos para que el alumnado decida cuáles les gustan más. Por ejemplo, que tengan que elegir una opción entre diferentes deportes colectivos 0 individuales, 0 entre diferentes juegos alternativos 0 tradicionales, o entre diferentes actividades en la naturaleza. También se puede dar a elegir entre dos actividades dirigidas de mejora de la condición física aérobica de las que se imparten habitualmente en un centro de fitness. Incluso en el propio cuaderno/libro de educación física del alumno, se pueden plantear dos actividades con el mismo fin, de manera que el estudiante sólo tenga que hacer la que más le guste o le llame la atención, pudiéndose convertir en un pasatiempo similar a los cuadernos de actividades que hacen los niños en verano. 
3) Reglas de un juego: Los estudiantes pueden inventar sus propios juegos o incluso proponer normas dentro de los juegos propuestos por el profesor. Por ejemplo, en un juego colectivo en el que el grupo debe conseguir dar diez pases sin que el equipo contrario robe la pelota, se puede establecer que en cada punto un equipo decida el tipo de pase que se tiene que dar.

4) Espacio: Si disponemos de diferentes instalaciones podemos darles a elegir dónde trabajar. También, si organizamos excursiones o rutas senderistas, les podemos dar diferentes posibilidades y que elijan la que más les guste. Del mismo modo, se puede pedir opinión al alumnado a la hora de limitar el espacio en las diferentes tareas planteadas, como por ejemplo en un partido de voleibol $3 \times 3$, donde pueden elegir la longitud y anchura de la cancha.

5) Tiempo: Otra variable sobre la que podemos trabajar para ceder autonomía es el tiempo. A la hora de plantear tareas podemos implicar a los estudiantes en la decisión de cuánto tiempo quieren que duren.

6) Material: Por ejemplo, se puede dar a elegir entre un balón de balonmano o uno de baloncesto para un juego colectivo de pases; o bien entre unas mancuernas, unas gomas o la propia resistencia de un compañero, en una tarea encaminada al trabajo de la fuerza. Igualmente resulta muy motivante la autoconstrucción de materiales para su empleo en diferentes unidades didácticas de juegos deportivos, alternativos y tradicionales (Fernández-Río y Méndez-Jiménez, 2012). También, dentro de un modelo disciplinario de adiestramiento (Del Villar, 2001), consistente en el establecimiento de rutinas organizativas, se puede implicar al alumnado en la colocación y recogida del material, estableciendo un sistema rotatorio de estudiantes y dándoles por tanto un rol autónomo en la gestión del material en la sesión.

7) Técnica de enseñanza y estrategia en la práctica: Se podría preguntar al alumnado si prefiere que para una determinada habilidad el profesor explique directamente la forma de ejecutarla (instrucción directa), o bien quiere que se plantee una secuencia didáctica de indagación. Lo mismo es aplicable para la estrategia en la práctica. Se puede preguntar a los estudiantes si prefieren trabajar un determinado contenido por partes (de una forma más analítica) o bien de forma jugada o global.

8) Normas de comportamiento: Resulta muy útil aplicar un modelo disciplinario de dinámica de grupos (Del Villar, 2001), en el que el profesor de educación física dedique las primeras sesiones del curso a establecer las normas de forma consensuada con el alumnado, de tal manera que consiga un mayor compromiso del estudiante con las conductas responsables.

9) Evaluación: El estudiante puede intervenir en el diseño de los instrumentos de evaluación, en los porcentajes asignados, e incluso en el mismo proceso, evaluando a los compañeros (coevaluación) o autoevaluándose.

Explicar los objetivos didácticos de cada actividad: Aunque algunos puedan pensar que es una pérdida de tiempo, la investigación ha demostrado claramente que es fundamental explicar lo que se persigue al plantear una determinada actividad, sesión o unidad didáctica. El hecho de que el estudiante sepa por qué tiene que realizar algo, le hace sentirse menos controlado y más autónomo. Si el docente de educación física plantea una tarea sin decirle al alumno lo que se trabaja en ella, y por qué puede ser importante realizarla, va a generar una sensación de imposición, sobre todo en aquellas tareas con las que el alumnado no disfrute. Por ejemplo, no es lo 
mismo que al estudiante se le mande hacer 30 abdominales sin ningún tipo de explicación, que se le indiquen los beneficios que tiene el acondicionamiento de la musculatura del tronco para la salud, centrados en la estabilidad del raquis y la resistencia abdominal. Igualmente, no es lo mismo que en un juego colectivo relacionado con el fútbol, se obligue a dar solamente un toque al balón, sin ninguna explicación, que se indique que esta limitación se establece para mejorar la circulación de balón, el desmarque y la ocupación de espacios libres. En la segunda situación de los dos ejemplos anteriores, el estudiante puede entender por qué se toman ciertas decisiones que afectan a la estructura de las tareas y las sesiones, se siente menos controlado, y su motivación autodeterminada, actitud y compromiso con la práctica va a ser mayor.

No crear dependencia de las recompensas: Aunque hemos mencionado que el feedback es muy importante para fomentar la sensación de competencia, el docente no debe abusar de él. El feedback es más necesario en las primeras fases del aprendizaje y luego el alumno tiene que aprender a pedirlo. Al fin y al cabo, las clases de educación física persiguen la práctica de actividad física autónoma y el estudiante no debe necesitar constantemente la presencia del profesor para llevar a cabo lo propuesto. Además, hay que tratar de evitar que las conductas se realicen sólo para obtener una determinada recompensa, como jugar un partido al final de la sesión. Se pueden utilizar recompensas de vez en cuando, pero no puede ser una tónica generalizada, pues ya hemos comentado que la regulación externa se asocia con consecuencias más negativas.

Utilizar estilos de enseñanza participativos: Los estilos participativos (Delgado Noguera, 1991), que ceden roles docentes al alumnado, son la máxima representación de la autonomía en la clase de educación física. Dentro de estos estilos encontramos la enseñanza recíproca, los grupos reducidos, y la microenseñaza. En la enseñanza recíproca se cede el rol de observador/corrector al estudiante, de tal manera que un compañero o compañeros ejecutan y otro les da feedback (e.g., trabajo de pases por parejas en voleibol). Es muy importante una adecuada y progresiva formación para la observación, así como facilitar una hoja de observación con pocos ítems (para no saturar de información) pero definidos con precisión. El trabajo por grupos reducidos parte de la misma dinámica, pero con un mayor número de roles docentes (e.g., anotador, ayuda, encargado de material, encargado de filmar, entrenador, etc.). También se puede utilizar un estilo de microenseñanza (rol docente completo) en el que el estudiante pueda dirigir en etapas menos avanzadas la fase inicial de la sesión, y en los últimos cursos sea capaz de dirigir la sesión completa, por ejemplo elaborando un programa de entrenamiento orientado a la salud para el desarrollo de una o varias cualidades físicas.

Utilizar estilos de enseñanza basados en la técnica de indagación: Los estilos cognitivos (descubrimiento guiado y resolución de problemas) y el creativo (Delgado Noguera, 1991), también proporcionan una gran autonomía al estudiante, pues éste tiene que buscar la/s forma/s más efectiva/s de resolver el problema planteado. Es muy importante que el profesor establezca una secuencia didáctica de preguntas que lleven al alumnado a descubrir la única respuesta eficaz en el caso del descubrimiento guiado, varias soluciones eficaces en el caso de la resolución de problemas, o todas las soluciones posibles dando rienda suelta a la creatividad. Resulta conveniente durante el proceso de indagación que la puesta en común de las soluciones se haga previamente en grupos pequeños para conseguir la implicación activa de todos lo estudiantes, y luego se consensue en gran grupo con el profesor.

Un ejemplo de descubrimiento guiado sería parcelar el gesto técnico del saque de tenis en voleibol en diferentes preguntas: ¿Se lanza el balón hacia arriba con una o 
dos manos? ¿La altura del lanzamiento es alta, media o baja? ¿Se golpea el balón con el brazo totalmente extendido por encima de la cabeza, sólo ligeramente extendido por encima de la cabeza, o a la altura de la cabeza? ¿Se golpea el balón por delante del cuerpo, por detrás o en la vertical? ¿En el armado del brazo hay una flexión completa del codo, semi-flexión o no se flexiona?

Un ejemplo de resolución de problemas sería plantear una secuencia de situaciones motrices encaminada a conocer el desmarque de ruptura y apoyo en fútbol. En la primera tarea se colocan dos atacantes y un defensor pasivo (no sigue al atacante). Uno de los atacantes debe desmarcarse y el otro le envía el balón. El atacante que se desmarca aprende que puede desplazarse en cualquier dirección y a cualquier velocidad, ya que el defensor no le sigue. Repetimos la situación con el defensor activo, y el atacante se da cuenta de que debe desplazarse a máxima velocidad. Por último, añadimos un defensor más y un atacante más, de manera que el atacante que se desmarca se da cuenta de que no le sirve desplazarse de forma paralela a la defensa, ya que ésta báscula, sino que tiene que hacer desmarques de ruptura en profundidad para recibir el balón, o de apoyo para crear un hueco en la defensa que pueda ser aprovechado por otro compañero.

Por último, un ejemplo de estilo creativo sería darle al alumnado una lista de cosas (belén, árbol de navidad, zoo, autobús, fórmula uno, cuarto de baño, etc.) para representar en grupo con todo el material deportivo que quiera.

\subsection{Estrategias para fomentar la relación:}

\section{a) Relación entre alumnos}

Fomentar la interacción grupal: Como ya se ha comentado anteriormente, la educación física, por sus características peculiares de interacción durante el movimiento, es la asignatura que permite fomentar en mayor medida las relaciones sociales entre los alumnos. Resulta de interés plantear tareas cooperativas con un objetivo común, en las que todos los miembros del grupo sean necesarios para conseguirlo. Del mismo modo, el profesor de educación física puede jugar con los agrupamientos para mejorar las relaciones. En esta línea, la aplicación de un estilo socializador (Delgado Noguera, 1991) en algún momento del curso (por ejemplo al inicio, cuando el grupo no se conoce) puede ser de gran utilidad. A continuación se describen algunos ejemplos de tareas que se ajustan a los principios del estilo socializador:

- Carrera por grupos situados en fila que tienen que pasarse un balón con las manos de delante hacia atrás (bien por encima de la cabeza, bien por debajo de las piernas), de manera que cuando recibe el último jugador corre con el balón en las manos a colocarse en primera posición y lo pasa de nuevo hacia atrás.

- Los alumnos se colocan en dos hileras enfrentadas, agarran las manos del compañero de enfrente y forman una red. Un alumno se lanza a esa red y es manteado hasta el final de la misma.

- Los alumnos forman un círculo agarrados por las manos y mirando hacia fuera. Deben desplazarse siguiendo las indicaciones del profesor sin soltarse y sin cerrar el círculo, como si fueran una única persona. 
Una estrategia interesante para ir cambiando los grupos durante una sesión socializadora, es repartir una carta de la baraja española e ir agrupando por palos y por números, aumentando el número de componentes progresivamente (e.g., por parejas, espadas con bastos, y oros con copas; igual pero espadas con oros y bastos con copas; ahora en grupos de cuatro, todos los del mismo número; Ahora grupos de diez, todos los del mismo palo, etc.).

Utilizar formas de agrupación flexibles y heterogéneas: Durante la clase de educación física, el docente no va a tener siempre como objetivo socializar. Por ello, la clave de los agrupamientos radica en combinar los diferentes sistemas existentes. Unas veces el profesor hará los grupos al azar, otras veces los hará equilibrados, otras juntará a los menos capaces con los más capaces para que se enseñen mutuamente, y otras veces dejará al alumnado agruparse de forma libre para juntarse con sus mejores amigos. Para que se dé un buen desarrollo social es necesario no abusar de ningún sistema de agrupamiento e ir alternándolos. La película Monstruos University, nada más comenzar, muestra muy claramente el problema de utilizar siempre un sistema de agrupamientos libres. Al bajar del autobús durante una excursión, la profesora indica que los alumnos se pongan por parejas. Mike Wazowsky (el pequeño monstruo verde con un solo ojo) empieza a preguntarle a varios de sus compañeros si se quieren poner con él. Todos le ignoran, incluso su primo que ni siquiera le reconoce y eso que muchas veces van en coche juntos. Al final Mike acaba, como de costumbre, formando pareja con la profesora. Sirva este ejemplo de reflexión para que el docente de educación física valore muy bien el aspecto relacional a la hora de establecer los grupos de trabajo.

Evitar la rivalidad entre compañeros y desarrollar el autocontrol: Ya hemos comentado con anterioridad que el profesor de educación física debe desarrollar una competencia sana, focalizada en la superación personal, el desarrollo de actitudes de deportividad y la adquisición de valores. No obstante, pueden surgir conflictos entre los estudiantes durante la realización de las tareas. Es importante educarles en el autocontrol para que no hagan algo (insultos o agresión al compañero) de lo que se puedan arrepentir o que pueda generar problemas. Es muy interesante trabajar con ellos el símil de la lata de coca-cola. Si una lata de coca-cola se nos cae al suelo y la abrimos nada más caerse, el líquido sale despedido y nos salpica. Sin embargo, si esperamos unos instantes podremos abrir la lata sin mojarnos. El caso de la lata de coca-cola sería similar a un problema que pueda surgir con un compañero. Si nada más tener el problema con el compañero me dejo llevar, puede que le acabe insultando o pegando. Sin embargo, si espero un poco y me calmo, podemos resolver el conflicto de una manera mejor.

\section{b) Relación profesor-alumno}

Crear un clima de preocupación y ser inteligente emocionalmente: El concepto de clima de preocupación (Gano-Overway y Guivernau, 2014) hace referencia a preocuparse por el alumnado más allá de la propia aula. El docente, además de enseñante de un determinado contenido, es un educador que influye en la vida de las personas. Resulta fundamental que el profesor se interese por las cuestiones y problemas de la vida cotidiana del estudiante, manteniendo una relación cercana pero sin perder el estatus. Se trata de representar una fuente de confianza y apoyo para el alumnado, preguntándole de vez en cuando por sus amigos, su familia, sus actividades fuera de la escuela (deportivas y no deportivas), su evolución en el resto de asignaturas, etc. El modelo disciplinario de desarrollo personal y social (Del Villar, 2001), que trata de identificar las causas de las conductas disruptivas, conociendo bien las circunstancias vitales que rodean al alumnado, se ajusta también muy bien a esta idea. Por supuesto, el docente debe aprenderse el nombre de todos los alumnos, 
dirigiéndose a ellos de una forma personal. Resulta especialmente interesante que el profesor trabaje su inteligencia emocional, tratando de percibir las emociones de los alumnos y mostrando las suyas propias. Es conveniente que el docente muestre su lado humano; que los alumnos no le vean sólo como docente, sino también como persona. Además, hay que tratar de emocionar a los alumnos, ya que lo que se aprende con emoción nunca se olvida (Cury, 2007).

Mostrar pasión: Uno de los elementos más importantes de la actuación docente, es demostrar pasión por lo que se hace. Está demostrado que la pasión se contagia, se transmite, tanto por un mecanismo psicológico, como por un mecanismo fisiológico relacionado con las neuronas espejo. Si veo una cara sonriente, sonrío. Si veo una cara malhumorada, me pongo de mal humor. La pasión armónica (no obsesiva) se ha asociado con las emociones y el afecto positivo, una mejor calidad de las relaciones personales y una mayor satisfacción con la vida (Vallerand, 2012). Los estudiantes perciben la pasión del docente y ésta les estimula a implicarse en mayor medida en lo que hacen.

Tener en cuenta la importancia motivacional de cada sesión: El modelo jerárquico de la motivación intrínseca y extrínseca (Vallerand, 2007) establece que la motivación situacional (en una tarea, conjunto de tareas o sesión) influye de manera significativa en la motivación contextual (hacia la educación física). Por ello, es fundamental que el profesor de educación física valore cada uno de los momentos en los que interactúa con los estudiantes, ya que todos esos momentos son relevantes motivacionalmente. Un docente puede tener uno o varios días malos por problemas personales, pero al entrar en el aula, como profesional que es, debe hacer el esfuerzo de dejar los problemas fuera y no pagarlo con los estudiantes. Dice un refrán que lo malo cuenta siete veces más que lo bueno, por lo que una actuación injusta ante los alumnos como consecuencia de los problemas personales del profesor, puede costarle no ser aceptado ya por ellos, aunque luego quiera cambiar de actitud.

\section{EL MODELO DE EDUCACIÓN DEPORTIVA Y SU RELACIÓN CON LA SATISFACCIÓN DE LAS NECESIDADES PSICOLÓGICAS BÁSICAS}

Teniendo en cuenta que el modelo de educación deportiva es un modelo de enseñanza bastante actual que trata de unir el contexto educativo y el contexto del ocio, a continuación describiremos brevemente los componentes del modelo y su asociación con la satisfacción de las necesidades psicológicas básicas, demostrada en un estudio reciente (Méndez-Giménez, Fernández-Río, y Méndez-Alonso, 2015). El modelo de educación deportiva (Siedentop, 1994) trata de trasladar la realidad del deporte competitivo al aula de educación física, pero con un tratamiento claramente educativo. Se busca establecer un nexo de unión entre la clase de educación física y la práctica deportiva que tiene lugar más allá del aula. Desde el inicio de la unidad didáctica se establecen equipos con diferentes roles: capitán, estadístico, observador, árbitro, entrenador, mánager, etc. Hay una primera fase de aprendizaje de habilidades técnicas y tácticas, que finalmente va orientada a la celebración de una competición deportiva entre los diferentes equipos.

El modelo presenta unas características particulares que ayudarían a satisfacer las necesidades psicológicas básicas. En relación a la necesidad de competencia, cabe reseñar que los juegos deportivos se suelen presentar en el modelo en sus formas reducidas o modificadas, lo que permite adaptarse a las características del 
alumnado, de manera que éste pueda sentirse competente. Además, para evitar la presencia de deportes con hegemonía masculina, se pueden introducir deportes alternativos como el ultimate (disco volador), de manera que las chicas tengan las mismas oportunidades de sentirse competentes que los chicos. Las unidades didácticas, concebidas como temporadas, tienen una duración larga (de al menos 12 sesiones), lo que facilita que los alumnos puedan aprender las habilidades requeridas y experimentar el éxito. También, el hecho de existir diferentes roles permite que cada estudiante pueda encontrar el rol que se le da mejor y, por lo tanto, pueda sentirse competente en el desarrollo de esa función. Igualmente, el hecho de existir un rol de observador que registra el rendimiento y aporta feedback, ayuda a los compañeros a conocer cómo lo han hecho y mejorar progresivamente. La existencia de varios premios (tanto individuales como colectivos, relacionados tanto con la victoria como con el juego limpio) permite que la mayoría de estudiantes obtengan un reconocimiento durante la práctica. La utilización de este modelo en educación física supone algo novedoso para el alumnado, ayudando a satisfacer su deseo innato de explorar y de lograr superar con éxito nuevos retos. Diferentes estudios revelan que el modelo de educación deportiva mejora el desarrollo de habilidades y la conciencia táctica (Méndez-Giménez, 2014).

Respecto a la necesidad de autonomía, podemos afirmar que el modelo es claramente autónomo, puesto que hay una cesión de responsabilidades docentes que fomenta la participación del alumnado a través de la adquisición de diferentes roles. De hecho, en el modelo se ponen de manifiesto estilos de enseñanza participativos como los grupos reducidos, al adquirir cada uno de los componentes del equipo un rol diferente, o la microenseñanza, cuando el alumno-entrenador tiene que dirigir las sesiones. Además, los estudiantes pueden elegir su equipación, grito o coreografía de guerra, y cómo se organizan durante la práctica. El modelo está orientado a establecer un nexo entre el aula de educación física y la competición deportiva real, por lo que tiene una vinculación clara con el fomento de la práctica de actividad deportiva extraescolar de forma autónoma.

La relación con los demás también se puede ver claramente favorecida por varios motivos. En primer lugar, se suele mantener siempre el mismo equipo, con sus señas de identidad, lo que genera un sentimiento de afiliación. Esto fomenta la interacción grupal, ya que los alumnos necesitan cooperar, ayudarse, coordinarse y aceptar cada uno su rol para conseguir un objetivo común. Además, el hecho de competir con otros equipos favorece la interacción con los demás, teniendo en cuenta que prima el juego limpio y el desarrollo de valores. Es importante destacar que este modelo pretende mostrar los valores educativos de la competición, reconociendo el esfuerzo y la superación personal, y evitando la rivalidad, de manera que se premia el respeto a las normas y la deportividad. La celebración de la clausura de la temporada con una fiesta con música, trofeos o fotos, también ayuda a mejorar las relaciones sociales del aula.

Aunque este modelo es interesante, el docente de educación física debe tratar de evitar comportamientos sociales negativos resultantes del ambiente competitivo generado. Si el modelo se aplica correctamente, teniendo en cuenta todas las pautas para que resulte educativo, está demostrado que ayuda a la inclusión, cohesión, liderazgo, cooperación, empatía y autodisciplina (Méndez-Giménez, 2014).

En un estudio realizado con estudiantes norteamericanos (Wallhead, Hagger, y Smith, 2010) se analizaron los efectos motivacionales de la aplicación del modelo de educación deportiva en clases de educación física. Se desarrollaron dos unidades didácticas, de rugby tag y bádminton, siguiendo cada una de ellas la siguiente estructura compuesta por 12 sesiones: Selección de equipos y roles (sesiones 1-2); 
Desarrollo de habilidades dirigidas por el profesor (sesiones 3-4); Fase de enseñanza entre iguales de pretemporada (sesiones 5-9); Torneo formal (sesiones 10-12). Tras la intervención se observó un incremento en la motivación autodeterminada hacia la educación física que predijo positivamente la motivación autodeterminada hacia la actividad física de ocio y un incremento de la actividad física realizada durante los recreos. Este estudio muestra cómo el modelo de educación deportiva podría influir en el desarrollo de una motivación más positiva y un mayor compromiso con la práctica de actividad física.

\section{CONCLUSIONES}

En este artículo se ha pretendido hacer un repaso de las estrategias motivacionales presentes en la literatura, pero haciendo un claro esfuerzo de adaptarlas con ejemplos concretos al aula de educación física. Se ha tratado de darle un giro a la didáctica de la educación física, introduciendo una vertiente motivacional, emocional y pasional, fruto de la revisión de la literatura científica. La didáctica se ha construido en nuestro país fundamentalmente a través de la experiencia, pero a día de hoy se empiezan a comprender los mecanismos científicos explicativos de los conceptos introducidos muchos años atrás, relacionados con la información inicial, el feedback, la organización y el control del aula, el aprovechamiento del tiempo de práctica, o las técnicas y estilos de enseñanza. Este artículo ha pretendido mostrar la asociación de todos estos conceptos con el constructo que determina la dirección, intensidad y persistencia de las conductas de los estudiantes: la motivación. Existen múltiples maneras para fomentar las necesidades psicológicas básicas del alumnado, mejorando su motivación autodeterminada y logrando consecuencias más positivas como el aprendizaje, el disfrute y la adherencia a la práctica deportiva. La literatura muestra que el modelo de educación deportiva puede ser una de estas maneras. Espero con esta publicación haber contribuido con un granito de arena a mejorar un ámbito que me apasiona, el de la enseñanza de la actividad física y del deporte. Espero lector, que los múltiples ejemplos que se han puesto, sean una fuente de estimulación para que a ti se te ocurran muchos más, adaptados a la realidad de tu aula.

\section{REFERENCIAS}

Beltrán-Carrillo, V. J., Devís-Devís, J., Peiró-Velert, C., y Brown, D. H. K. (2012). When physical activity participation promotes inactivity: Negative experiences of Spanish adolescents in physical education and sport. Youth and Society, 44, 327.

Cury, A. (2007). Padres brillantes, maestros fascinantes. Barcelona: Planeta.

Deci, E. L., y Ryan, R. M. (1985). Intrinsic motivation and self-determination in human behavior. New York: Plenum.

Deci, E. L., y Ryan, R. M. (2000). The "what" and "why" of goal pursuits: Human needs and the self-determination of behaviour. Psychological Inquiry, 11, 227-268.

Delgado Noguera, M. A. (1991). Los estilos de enseñanza en la educación física. Propuesta para una reforma de la enseñanza. Granada: I.C.E. de la Universidad de Granada. 
Del Villar, F. (2001): La función docente en la educación física. En Vázquez, B. (Ed.), Bases educativas de la actividad física y el deporte (pp. 199-226). Madrid: Síntesis.

Dweck, C. S. (1999). Self-theories: Their role in motivation, personality, and development. Philadelphia: Taylor and Francis.

Fernández-Río, J., y Méndez-Giménez, A. (2012). Innovative practices through the use of self-made materials. The cooperative learning model in Spain. En B. Dyson y A. Casey (Eds.), Cooperative learning in physical education: A research-based approach (pp. 42-56). London: Routledge.

Gano-Overway, L., y Guivernau, M. (2014). Caring in the gym. Reflections from middle school physical education teachers. European Physical Education Review, 20, 264-281.

Generelo, E., Julián, J. A., y Zaragoza, J. (2009). Tres vueltas al patio. La carrera de larga duración en la escuela. Barcelona: Inde.

González-Cutre, D., Martínez-Camacho, A., Gómez-Rijo, A., y Moreno, J. A. (2010). La motivación autodeterminada en la actividad física y el deporte: Propuesta de intervención práctica. En J. A. Moreno y E. Cervelló (Eds.), Motivación en la actividad física y el deporte (pp. 151-170). Sevilla: Wanceulen.

González-Cutre, D., y Moreno, J. A. (2009). Estrategias motivacionales para una transformación social a través de la actividad físico-deportiva. En J. A. Moreno y D. González-Cutre (Eds.), Deporte, intervención y transformación social (pp. 111-139). Río de Janeiro: Shape.

González-Cutre, D., Sicilia, A., Beas-Jiménez, M., y Hagger, M. S. (2014). Broadening the trans-contextual model of motivation: A study with Spanish adolescents. Scandinavian Journal of Medicine and Science in Sports, 24, e306-e319. doi: 10.1111 / sms.12142

González-Cutre, D., Sicilia, A., Sierra, A. C., Ferriz, R., y Hagger, M. S. (2016). Understanding the need for novelty from the perspective of self-determination theory. Personality and Individual Differences, 102, 159-169.

Hagger, M. S., y Chatzisarantis, N. L. D. (2012). Transferring motivation from educational to extramural contexts: A review of the trans-contextual model. European Journal of Psychology of Education, 27, 195-212.

Karageorghis, C. I. (2017). Applying music in exercise and sport. Champaign, IL: Human Kinetics.

Méndez-Giménez, A. (Ed.). (2014). Modelos de enseñanza en educación física. Madrid: Grupo 5.

Méndez-Giménez, A., Fernández-Río, J., y Méndez-Alonso, D. (2015). Modelo de educación deportiva versus modelo tradicional: Efectos en la motivación y deportividad. Revista Internacional de Medicina y Ciencias de la Actividad Física y del Deporte, 15(59), 449-466.

Moreno, J. A., Cervelló, E., González-Cutre, D., Julián, J. A., y Del Villar, F. (2011). La motivación en el deporte. Claves para el éxito. Barcelona: Inde. 
Moreno, J. A., y González-Cutre, D. (2006). A permanência de practicantes em programas aquáticos baseada na Teoría de Autodeterminação. Fitness \& Performance Journal, 5, 5-10.

Nicholls, J. G. (1989). The competitive ethos and democratic education. Cambridge, MASS: Harvard University Press.

Ntoumanis, N. (2012). A self-determination theory perspective on motivation in sport and physical education: Current trends and possible future research directions. En G. C. Roberts y D. C. Treasure (Eds.), Advances in motivation in sport and exercise (pp. 91-128). Champaign, IL: Human Kinetics.

Priest, D. L, y Karageorghis, C. I. (2008). A qualitative investigation into the characteristics and effects of music accompanying exercise. European Physical Education Review, 14, 347-366.

Ryan, R. M., y Deci, E. L. (2000). Self-determination theory and the facilitation of intrinsic motivation, social development, and well-being. American Psychologist, 55, 68-78.

Ryan, R. M., y Deci, E. L. (2017). Self-determination theory: Basic psychological needs in motivation, development, and wellness. New York: Guilford Press.

Siedentop, D. (1994). Sport education. Quality P.E. through positive sport experiences. Champaign, IL: Human Kinetics.

Vallerand, R. J. (2007). Intrinsic and extrinsic motivation in sport and physical activity. A review an a look at the future. En G. Tenenbaum y R. C. Eklund (Eds.), Handbook of sport psychology ( $3^{\mathrm{a}}$ ed., pp. 59-83). New York: John Wiley.

Vallerand, R. J. (2012). Passion for sport and exercise: The dualistic model of passion. En G. C. Roberts y D. C. Treasure (Eds.), Advances in motivation in sport and exercise (pp. 160-206). Champaign, IL: Human Kinetics.

Wallhead, T. L., Hagger, M., y Smith, D. T. (2010). Sport education and extracurricular sport participation: An examination using the trans-contextual model of motivation. Research Quarterly for Exercise and Sport, 81, 442-455. 